\title{
A Long Pulse High-Power Diode Based on a Microelectronic Emitter
}

Barry Marder, Collins Clark, Robert Walko, Jim Fleming

Prepared by

Sandia National Laboratories

Albuquerque, New Mexico 87185 and Livermore, California 94550

for the United States Department of Energy

under Contract DE-AC04-94AL85000

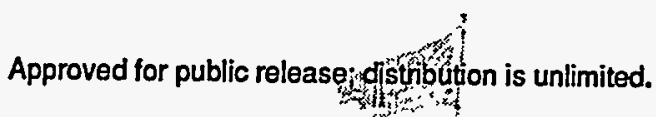

(n)

s.

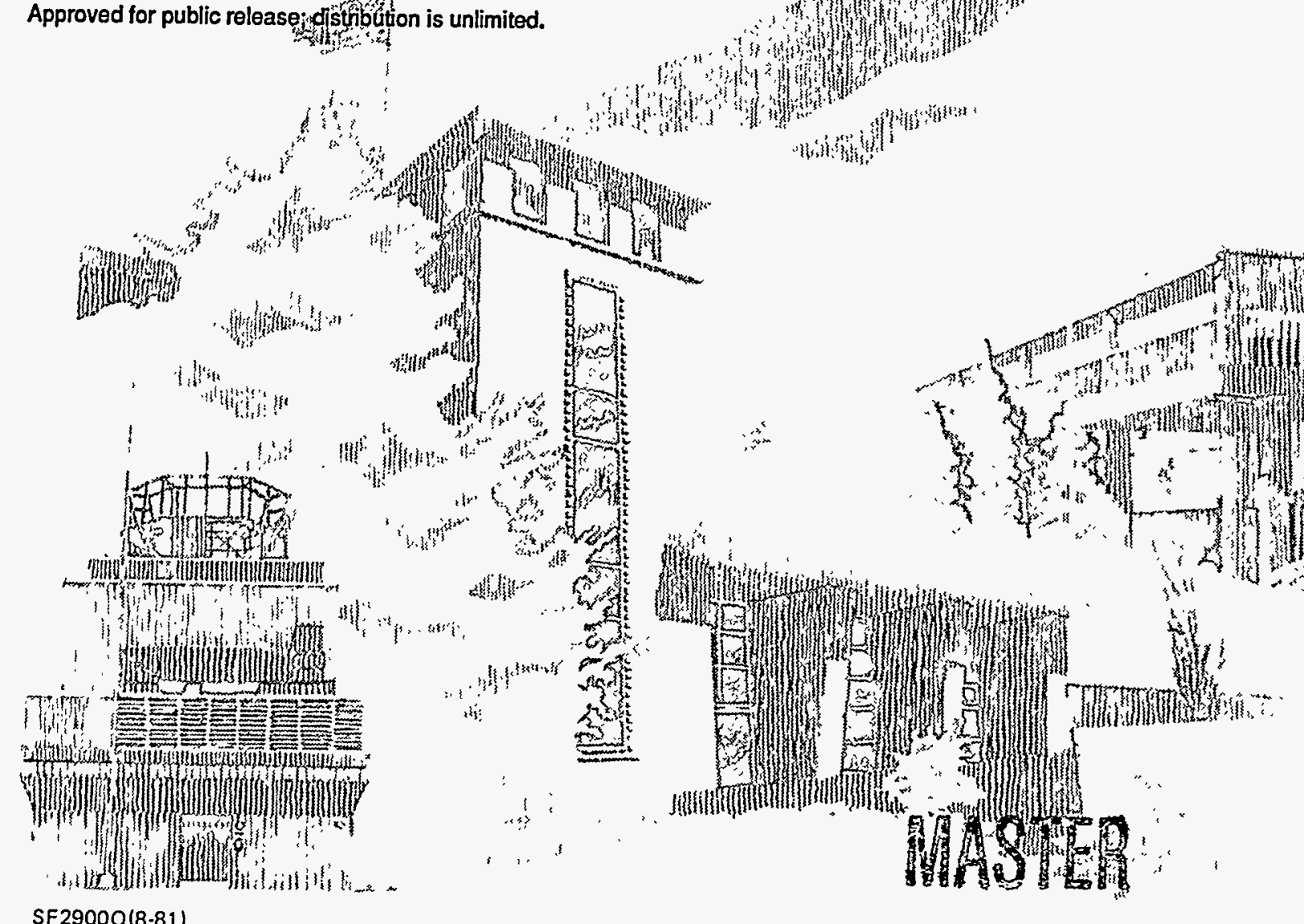


Issued by Sandia National Laboratories, operated for the United States Department of Energy by Sandia Corporation.

NOTICE: This report was prepared as an account of work sponsored by an agency of the United States Government. Neither the United States Government nor any agency thereof, nor any of their employees, nor any of their contractors, subcontractors, or their employees, makes any warranty, express or implied, or assumes any legal liability or responsibility for the accuracy, completeness, or usefulness of any information, apparatus, product, or process disclosed, or represents that its use would not infringe privately owned rights. Reference herein to any specific commercial product, process, or service by trade name, trademark, manufacturer, or otherwise, does not necessarily constitute or imply its endorsement, recommendation, or favoring by the United States Government, any agency thereof or any of their contractors or subcontractors. The views and opinions expressed herein do not necessarily state or reflect those of the United States Government, any agency thereof or any of their contractors.

Printed in the United States of America. This report has been reproduced directly from the best available copy.

Available to DOE and DOE contractors from

Office of Scientific and Technical Information

PO Box 62

Oak Ridge, TN 37831

Prices available from (615) 576-8401, FTS 626-8401

Available to the public from

National Technical Information Service

US Department of Commerce

5285 Port Royal Rd

Springfield, VA 22161

NTIS price codes

Printed copy: A03

Microfiche copy: A01 
SAND95-2061

Distribution

Unlimited Release

Category UC-411

Printed November 1995

\title{
A Long Pulse High-Power Diode Based on a Microelectronic Emitter
}

\author{
Barry Marder, Collins Clark, Robert Walko, Jim Fleming \\ Sandia National Laboratories \\ Albuquerque, NM 87185
}

\begin{abstract}
Microelectronic cathode emitter technology being developed at Sandia for supplying continuous low current for flat panel displays appears to be a promising technology for providing high currents when operated in a pulsed, higher voltage mode. If currents in excess of one amp per square centimeter could be produced for tens of microseconds at several kilohertz repetition rate, important applications in such as large volume food or waste sterilization, in situ detection, and high power microwave production could be achieved. A testbed was built to perform the experiments. The desired current densities have been demonstrated using small emitter arrays.
\end{abstract}


spacings down to sub $1000 \AA$. This is far smaller than that achievable using standard photolithography. Our initial experiments investigated whether simply an expanded version of this structure could met the requirements of this project.

\section{Results of Others}

There are many possible approaches to the fabrication of electron emission displays and emission tips. In order to meet the requirements of this project the emission from each tip (assuming a simple cubic packing of tips 5 microns on a side) is $0.25^{*} 10^{-6}$ amps per tip. The current density per tip for a variety of different tip types is given in Table 1 .

These results indicate that, a variety of different types of tips could potentially meet the requirements of this project. However, it should be noted that these results were obtained from small numbers of, or even single tips, and as will be seen later, most of the fabrication problems are the result of there being large numbers of tips.

The type of tip which has been most thoroughly investigated is that based on the approach developed by Spindt. This approach has been applied to both flat panel displays and field emission devices for RF applications. These devices are well characterized (though there are apparently many undocumented process "tricks") and are capable of high current densities averaged over small number of tips. The general approach is well documented in the literature and will not be discussed in detail in this report. In short, the emitter-to-gate tip spacing is determined by either photolithography or other more novel approaches (submicron holes are desirable). The tip is formed using a combination of electron beam deposition and lift-off techniques. At present, the tool sets required for uniform and unidirectional electron beam deposition over large areas are not widely available. Because of this drawback we did not chose to pursue this approach.

There are numerous other approaches, sharp silicon tips formed by reactive ion or wet etching, metal wedge emitters, lateral emitters, diamond emitters, porous silicon emitters and so on.

However, none of these approaches has been demonstrated to be clearly superior, nor has any one of them been demonstrated to come close to meeting the requirements of this project. Because of this and the problems inherent in the Spindt approach we felt that the best course of action was to first investigate whether or not our technology could be applied to this project.

\section{Fowler-Nordheim Behavior}

Electron filed emission processes are described by the Fowler-Nordheim expression:

$$
\mathrm{J}=\mathrm{AE} \mathrm{E}^{2} \exp \left[-\mathrm{B}\left(\Phi^{3 / 2} / \mathrm{E}\right)\right]
$$

where $J$ is the emitted electron current density, $A$ and $B$ are constants, $E$ is the electric field, and $\Phi$ is the work function of the emitter. This expression clearly shows the very strong dependence of the electron current density on the field and work function of the emitter tip. The results of field emission testing are typically presented in the form $\ln \left(\mathrm{J} / \mathrm{V}^{2}\right)$ vs. $1 / \mathrm{N}$. This results in a straight line in the case of Fowler-Nordheim type emission. However, care must be taken not to assume that just because the data lie on a straight line with correct slope that this is the result of electron field emission in the case of structures where the collector is not physically separated from the emitter by external electronics. The reason for this is that various surface and bulk leakage mechanisms can result in high currents which have the same current/voltage characteristics as FowlerNordheim emission. All the results reported here were obtained from tests in which the collector is physically separated from the emitter array by a separate circuit. 
Initial Results

Initial tests were made on structures fabricated under a separate CRADA using a technology developed at SNL. Testing was performed by the industrial partner since they had the necessary fittings on hand and were familiar with the parts. The base pressure of the chamber was $\sim 10^{-7}$ Torr. Electrical connection was made to the parts with silver epoxy. Prior to testing the parts were backed overnight in the chamber at $\sim 200^{\circ} \mathrm{C}$. The part tested is similar in design to that shown in figure 3 . The emitter and the gate were both fabricated from in situ doped poly silicon of $\sim 400$ micro-Ohm am resistivity. In this structure a single pixel $\sim 300$ microns on a side was tested. While the part supplied enough current for its anticipated application, a high voltage phosphor flat panel display, as the gate voltage was ramped up the parts failed at current densities many of orders of magnitude lower than those required for this work. In fact it was found that even at low current densities, the parts eventually failed. Examination of the failed parts clearly showed that they had suffered extensive damage, as shown in figure 4 . These failures were eventually traced to arcs generated in the test cell.

There are at least three possible reasons for these failures, variations in tip sharpness, instability in the work function and a failure associated with the test system and not the emitters themselves. As can be seen from the Fowler-Nordheim equation, small changes in the field result in large changes in emitter current. Changes in sharpness along the emitter wedge give rise to large variations in emission current density. For low current density applications this is not a grave problem as long as there are a sufficient number of high sharpness sites within the desired area. However, as the voltage is increased, the current from these initially emitting regions increases rapidly to the point where the active emitters fail before the other sites become active. (The failure of electron emitters is widely seen, though the mechanism is unknown, it typically appears that the emitter melts and then explodes.)

A second possibility is that the failures are the result of the instability of the silicon emitters, especially during turn on. The instability of emission from silicon is clearly shown in figure 5 . Initially, the voltage must be increased to relatively high values before any emission of electrons is observed. The current then increases rapidly and the voltage must be rapidly reduced to prevent device burnout. We speculated that before emission begins the native oxide that forms on the silicon must first be broken down. However, once the native oxide breaks down, the field is so high that the resulting current density results in tip burn out. It may be that this susceptibility of the silicon emitters to oxide formation followed by the sudden breakdown of this oxide results in part failure.

A third possible reason for part failure is a sudden high voltage transient across the device due to a failure in some portion of the test chamber circuit. The most probable source of this high voltage is the $5000 \mathrm{~V}$ applied to the high voltage phosphor screen. At first this possibility was discounted, however, it now appears that chamber related failures are responsible for most of the failures observed to date. Failures like those shown in figure 4 have only been encountered only in the CRADA partners test chamber and not in the test chamber recently made available to us at SNLL (Felter, 8715). However, at the time the SNLL chamber was not available and this possibility was discounted and subsequent work was performed to try to address the first two issues: tip sharpness non-uniformity and silicon instability.

These initial failures lead us to the conclusion that it would not be possible to achieve the objective of this LDRD using a slight modification of our existing technology. Since this was determined relatively early in the FY the rest of our time was spent trying to develop a different approach to satisfy the project needs. 


\section{Micromachined Silicon Tip Structures Formed by KOH Etching}

We attempted to overcome the problem of emission non-uniformity due to variations in local sharpness through the implementation of a well defined tip structure. It was hoped that if the tip formation process was controllable enough, that sufficient uniformity of sharpness could be achieved that all the tips began to emit at essentially the same voltage. To achieve this end we developed a novel combination of $\mathrm{KOH}$ etching, masking and oxidation sharpening.

The use of $\mathrm{KOH}$ as a Si etchant is well know, while the actual mechanism of the process is unclear, $\mathrm{KOH}$ etches 111 planes at rates hundreds of times slower than other low index planes. $\mathrm{KOH}$ also etches $\mathrm{SiO}_{2}$ and $\mathrm{SiN}$ very slowly. This property is used to form pits in 100 type material bounded by 111 planes. The geometry of the pit is determined by the initial shape of the mask material. Once the 111 planes meet the etch process stops, ensuring excellent uniformity over large areas. Unfortunately, the situation of pyramidal tips bounded by 111 planes is more complicated. In this case, the tips sides are defined by 111 planes. However, the intersection of the planes is an exposed corner which is not a 111 plane. These exposed corners etch rapidly distorting the structure. Also, once the tip is formed, the exposed material is again not a 111 plane and the tip is therefore rapidly blunted. These two problems make it impossible to form large arrays of uniformly sharp tips over the large areas required by this project using standard $\mathrm{KOH}$ etching. For similar reasons, the standard approaches to this problem, organic additives and orientation to the faster etching, higher index, planes is also impractical.

The approach that we have taken to this problem is to first pattern a series of trenches parallel to the 111 planes using $\mathrm{KOH}$ etching and then pattern and define a series of orthogonal trenches to obtain a series of ribbed comb structures. Since this structure is formed by stopping of 111 planes on inside corners it can not be overetched and across the wafer uniformity should be excellent. The structures are then sharpened using oxidation sharpening. (Oxidation sharpening is based on the fact that sharp Si structures oxidize less rapidly than blunter structures due to stress changes in that occur during the oxidation process.) This results in a sharpening of already sharpened structures. This process is schematically shown in figure 6.

Results obtained using an unoptimized 3 micron line and space mask set are shown in figure 7 , which clearly shows that the approach can result in very sharp structures. However, the subsequent fabrication of a well defined gate structure along with the necessary contact pads proved to be a problem. Because of this, and due to greater promise and versatility offered by the metal wedge approach described below, this line of attack was abandoned.

\section{Metal Wedge Emission Structures}

The other approach which was investigated was the use of metal emitters in the from of either vertical wedges or combs of TiN or Ni on TiN. The use of a metal emitter offers a number of possible advantages. Firstly, neither TiN or Ni have the problems of native oxide formation that $\mathrm{Si}$ has, secondly, for reasons that are not well understood, metals consistently show a higher emission current density than silicon, Table 1.

The process which evolved is loosely related to the initial silicon wedge structure. The unique fabrication sequence which developed is illustrated in figure 8. An example of finished parts is given in figure 9. The emitter-to-gate separation in this case is determined by the thickness of a conductive film (in situ doped amorphous $\mathrm{Si}$ with a resistivity of $\sim 500 \mathrm{Ohm}-\mathrm{cm}$ ). The emitter wedge is a either TiN or Ni coated TiN supported by a plug of CVD deposited W. The wedge is 
exposed by chemical mechanically polishing the W and TiN down to the Si and then recessing the $\mathrm{W}$ and $\mathrm{Si}$ using a F plasma. (The etch rate of TiN in F is very low and the etch rate of Si is greater by roughly a factor of 4 than the rate of W-in F.) This opens up the spacing between the gate and emitter wedge and recesses the tungsten slightly to expose the TiN wedge. In some cases, a second orthogonal etch of the TiN/W structure was performed to create a comb emission structure. The gate is either TiN or a overhung SiN structure onto which is deposited $300 \AA$ Ni using electron beam evaporation. In this latter case the emitter is also coated with $\mathrm{Ni}$. The dielectric between the gate and the emitter is a stack of $3000 \AA$ of $S i N$ on top of $10000 \AA$ of thermal oxide.

The first two lots of parts using this design failed to produce working devices for reasons unrelated to the design itself. Scanning electron micrographs of the third lot did not reveal any obvious defects. However, when tested the parts were found to be partially shorted between the gate and the emitter. The resistivities of the $1 \mathrm{~cm}$ by $1 \mathrm{~cm}$ arrays was $10^{5}-10^{3} \mathrm{ohms}$. This is expected to result in an unacceptably high emitter-to-gate current. While this result seems to be at odds with the SEM analysis, it seems likely that over the very large extent of the array, defects will be present. The pitch of the structure is $\sim 2$ microns which results in a total emission wedge length of $\sim 100 \mathrm{~m} / \mathrm{cm}^{2}$. As a point of reference, the emission wedge density for a flat panel display is 1000 times less. Furthermore, in this design all the emitter wedges are interconnected through either a low resistivity metal line or over a very large area and therefore low resistivity poly silicon/single crystalline Si pathway. Thus a single conductive defect larger than the 0.3 micron gate-to-emitter spacing will short the entire device. However, it was possible to test a small portion of one of the devices, the I-V curve of which is shown in figure 10 . The turn on voltage was a low 30 volts and there was not the initial turn on instability observed with silicon emitter structures. However, these initial tests were also performed in the test chamber with the apparent arcing problem.

The shorting problem resulted in a further redesign of the mask design, instead of forming long emission wedges all of which are effectively electrically interconnected, we have designed a structure in which each emitter is a individual ring $~ 0.4$ microns in diameter. Each emitter ring is separated electrically one from the other by a resistive poly silicon layer. The value of the resistor in series with each emitter ring is $\sim 3 \mathrm{MOhms}$. Therefore, even in the event of a dead short between the gate and the emitter, the minimum resistivity will be $3 \mathrm{MOhms}$. This resistor between the back contact and the emitter wedge will also serve to limit the current from any part that is for any reason (low local work function or higher sharpness/local field) emitting at a far higher current density than other parts. For example, assuming a similar turn on behavior as the part in figure $10,30 \mathrm{~V}$, a 1 microamp current across the internal resistor will result in a $10 \%$ drop in field. This should "level" the emission between separate rings and prevent tip blow out. These parts have since been fabricated and tested at SNLL in a chamber where arcing between the collector and the emitter array is not a problem. Tests of a 100 tip array resulted in a maximum DC current density of 3 amps per centimeter squared averaged over the active portion of the device, Figure 11 . At the end of the test it was found that the phosphor screen had burned out. We speculate that the screen had outgassed as it failed and the resulting pressure increase initiated an arc which destroyed the device. The current density achieved in this test is in excess of that required by this program and both the literature and the experience of SNLL indicate that significantly higher current densities are achievable using a more rugged collector and pulsed operations.

This result clearly shows that sufficiently high current densities are achievable using metal edge devices. What is now required is that the arrays be made large enough that it becomes feasible to tile them to create the desired emission area.

Summary and Conclusions 
Initial attempts to adapt silicon based emission structures designed for field emission flat panel display applications to this project were unsuccessful. Though most of the early failures were the result of arcs caused by the test system, it seems performance of these devices at high current densities will be unacceptable due to surface instability and non-uniformity's in wedge sharpness. A novel process was developed which enabled the fabrication of very sharp and apparently uniform silicon tips. However, due to difficulties gating this type of structure and as a result of encouraging progress on metal wedge structures, this approach was dropped. A novel metal wedge emitter structure was developed and emission was demonstrated at reasonable voltages from small regions of these parts. However, the very long effectively continuous emitter-gate length $(100 \mathrm{~m}$ per $\mathrm{cm}^{2}$ ) coupled with a very emitter-to-gate spacing of 0.3 microns appears to have made the structures very sensitive to defects and shorts between the gate and the emitter. This problem in turn has been addressed by changing the design to that of a series of small emitter rings each in series with a integral current limiting resistor. Since the rings are only interconnected through the current limiting resistor, a short between any ring and the gate will have a minimal effect on the overall structure.

\section{Figures:}

Figure 1. Numerical simulation of micro-emitter showing equipotential surfaces and electron trajectories.

Figure 2. Schematic of the experimental apparatus.

Figure 3. Overview of the gated fillet fabrication process.

Figure 4. Example of post-testing damage typically observed at current densities far below those required by this project. The damage was eventually identified as resulting from the test cell design and not as a result of a failure associated with the part design.

Figure 5. Example of the inherent instability of silicon based emission structures. Voltages between the gate and emitter of about $100 \mathrm{~V}$ were required to break through the native oxide. However, once the oxide breaks down the current rapidly increases. (In this case the breakdown occurred as the voltage was being ramped down resulting in the apparent negative slope.) The system was computer controlled to shut down at currents higher than $150 \mathrm{nAmps}$. If the voltage was fixed at $100 \mathrm{~V}$ the part would have burned out.

Figure 6. Schematic of the silicon tip fabrication sequence.

Figure 7. Examples of sharp, uniform silicon tips formed by $\mathrm{KOH}$ etching and oxidation sharpening.

Figure 8 . The fabrication sequence for the formation of metal wedge emitters.

Figure 9. Scanning electron micrograph of a finished metal emitter part.

Figure 10. Current-voltage characteristic of a small section of a metal fillet emitter similar to that shown in previous figure.

Figure 11. Current-voltage characteristics of a metal edge device with 100 emitting sites on a 5 
micron close packed pitch. The average current density achieved is $3 \mathrm{~A} / \mathrm{cm}^{2}$. This is greater than the $1 \mathrm{~A} / \mathrm{cm}^{2}$ required by this project and could be increased by using a more rugged collector and pulsed operation. 


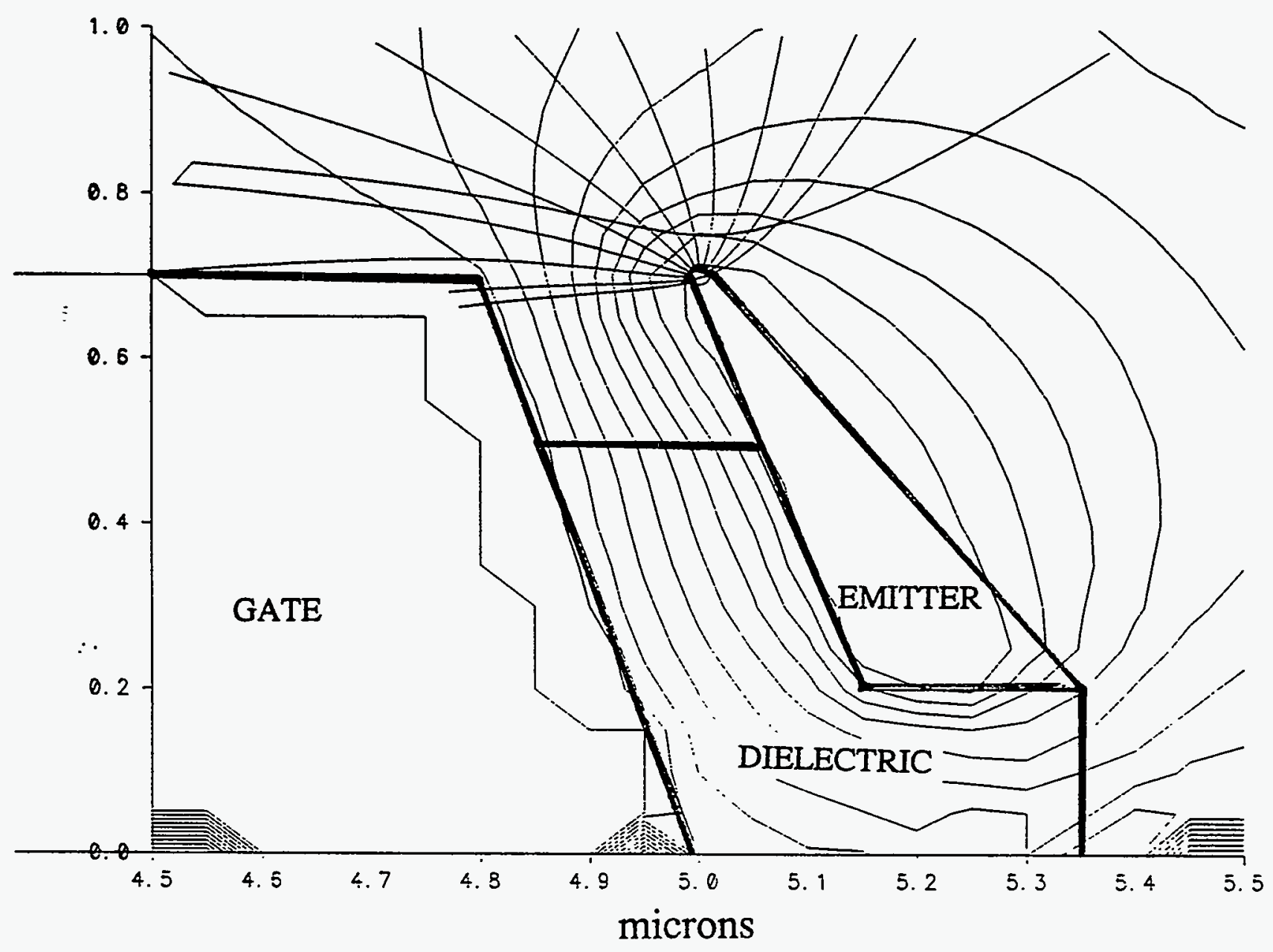

Figure 1

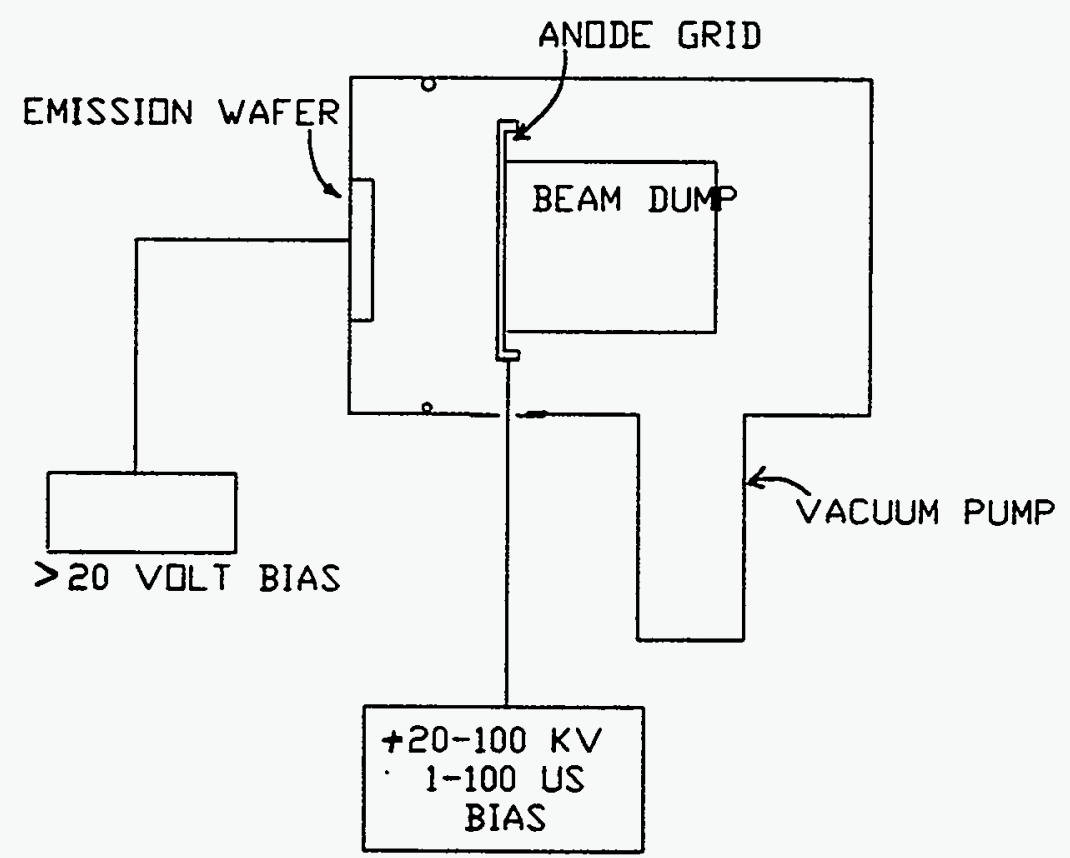

Figure 2

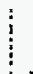


1) Deposit and pattern oxide.

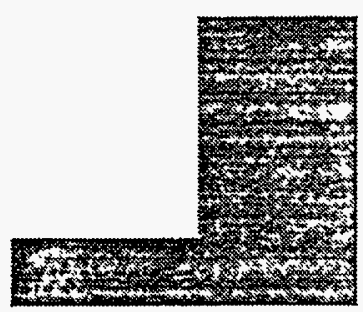

4) Deposit conducting emitter layer.

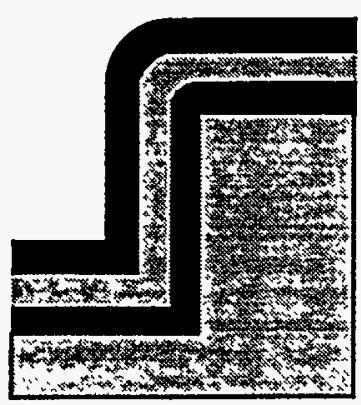

2) Deposit and pattern grid. 3) Deposit spacer oxide.
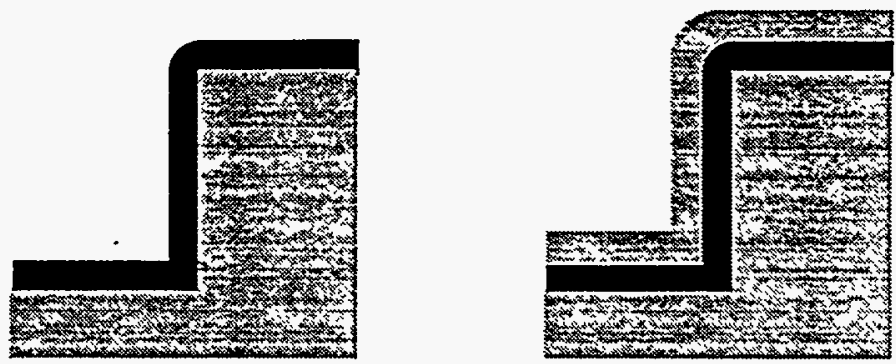

5) Form emitter wedge by RIE.

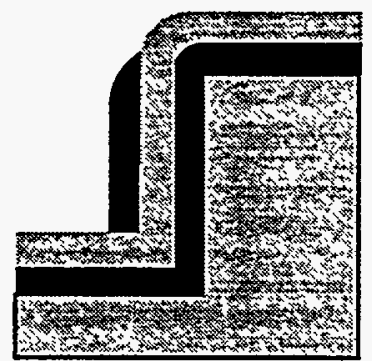

6) Partially remove oxide separator.

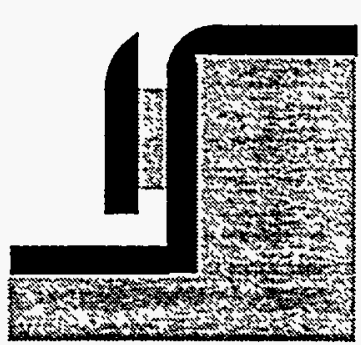

Figure 3 

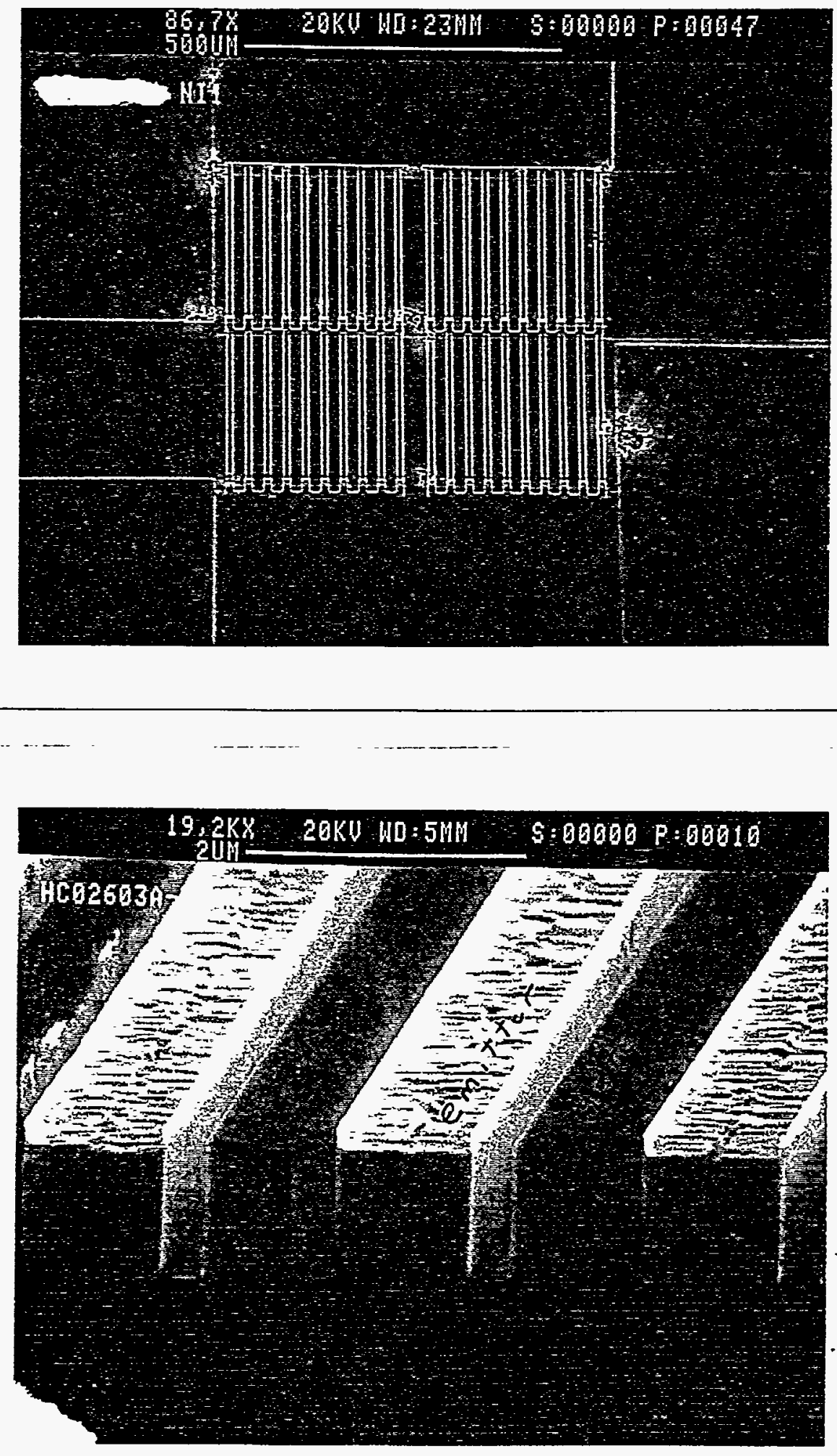

Figure 4 


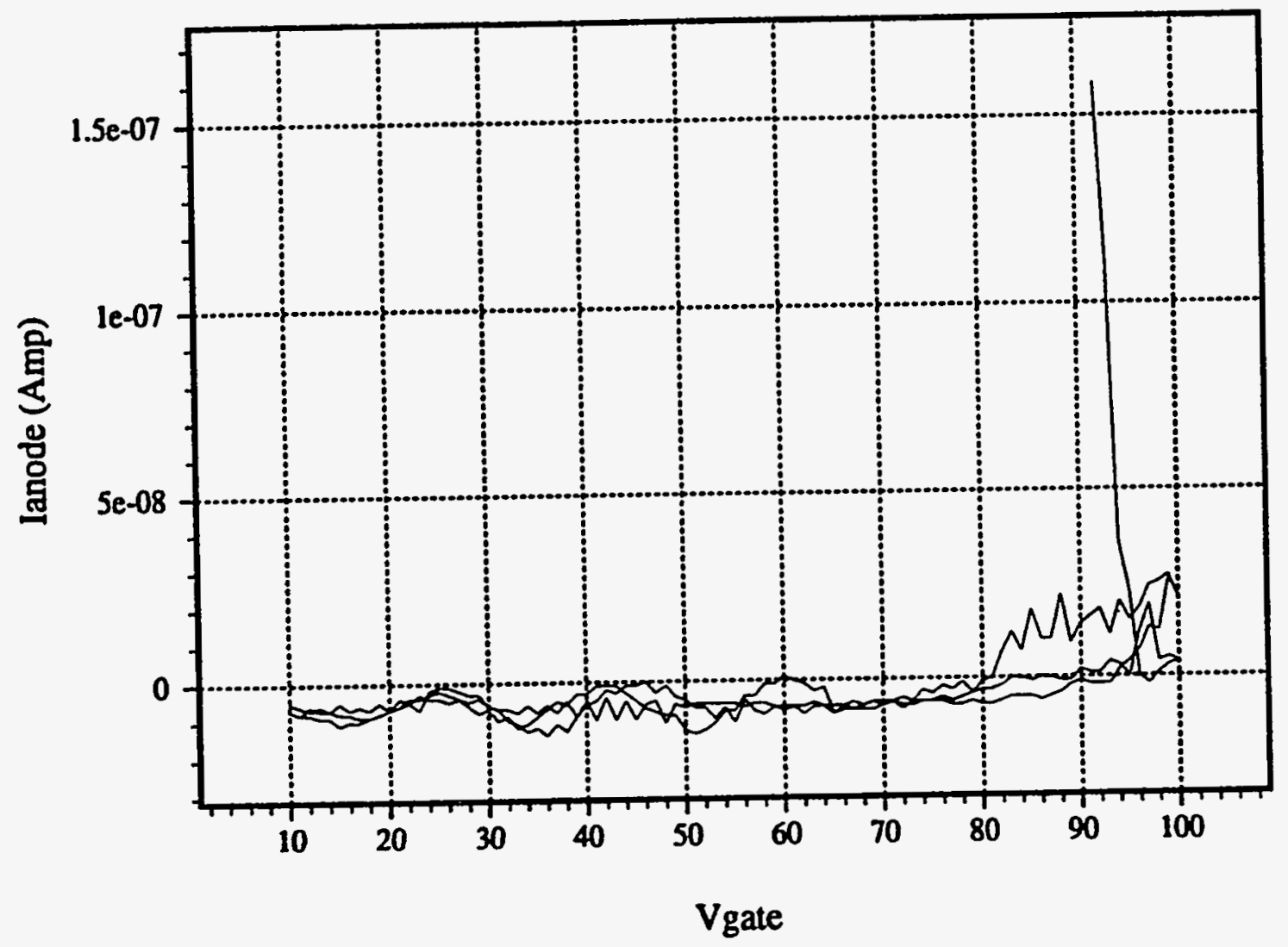

Figure 5 

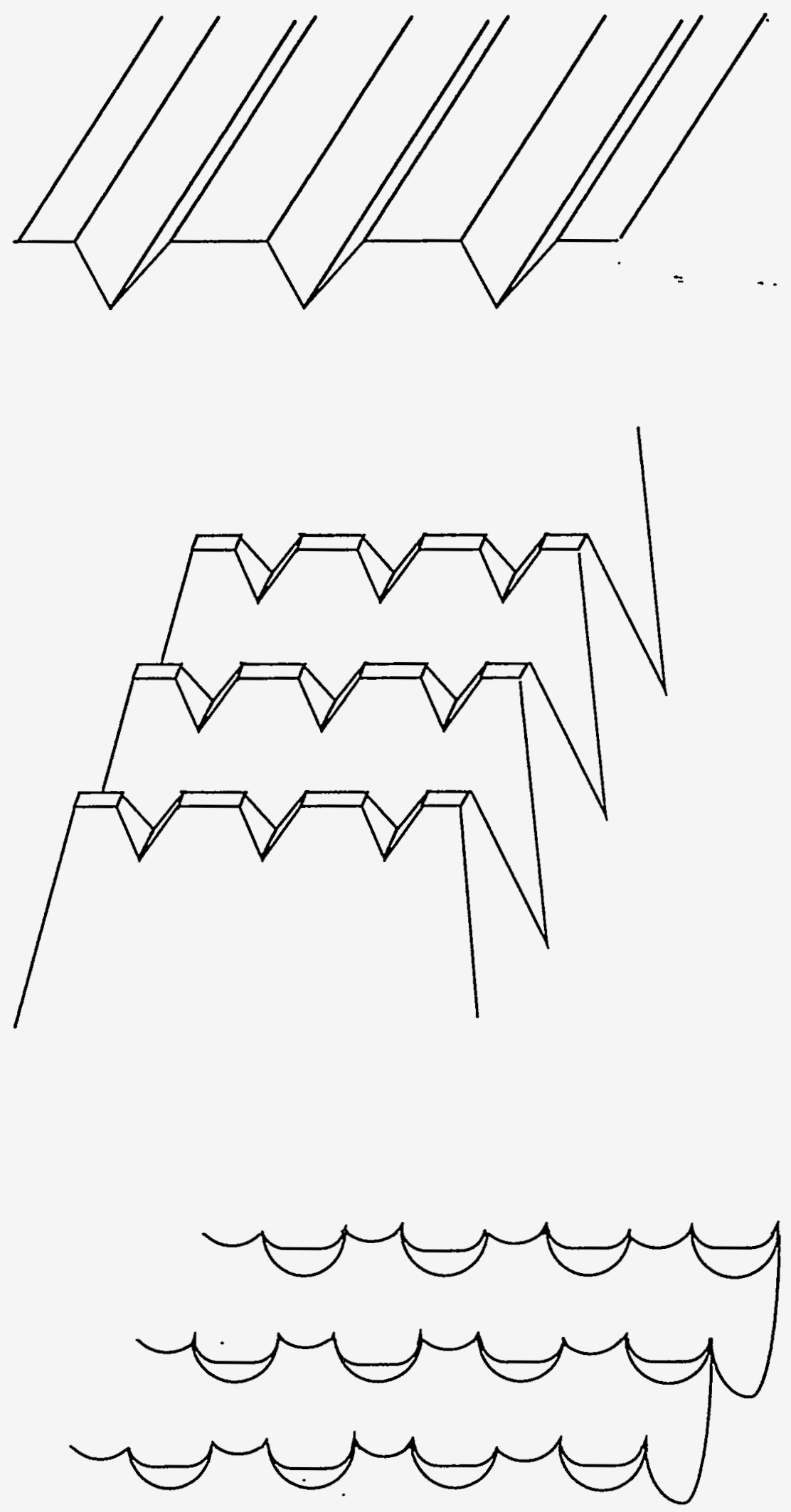

First deposit ixide and pattern it into strips along 110 directions. Then use the oxide as a mask as a $\mathrm{KOH}$ etch mask to create $\mathrm{V}$ grooves.
Repeat after the etch but pattern the second set of $\mathrm{V}$ groves perpendicular to the original set.
Oxidation sharpen and then strip the oxide. Repeat as needed to obtain the desired shape. 


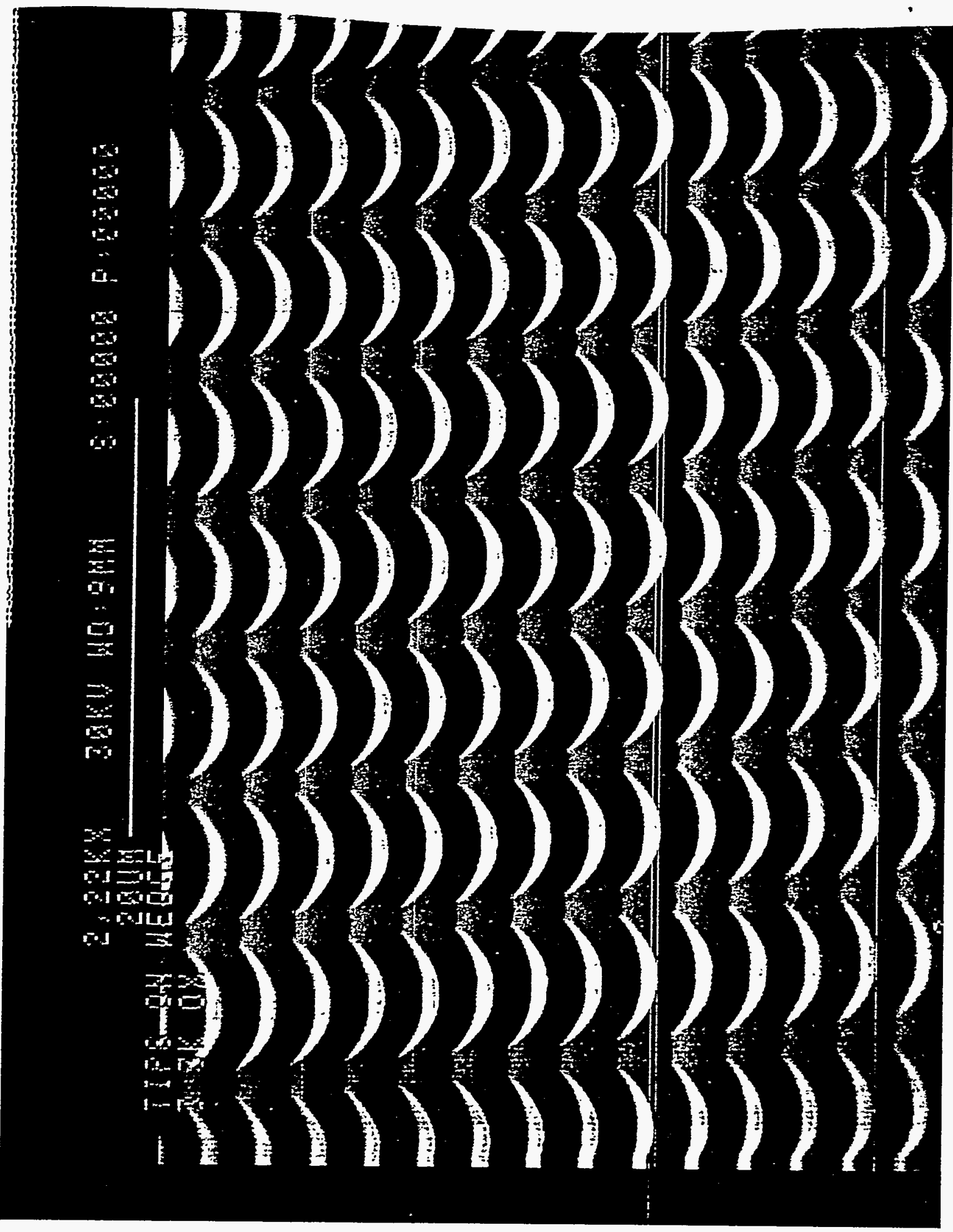

Figure 7 
Grow thermal oxide, deposit TiN, photo pattem, etch TiN, etch $\mathrm{SiO}_{2}$ down to Si substrate. This forms the TiN gate structure.

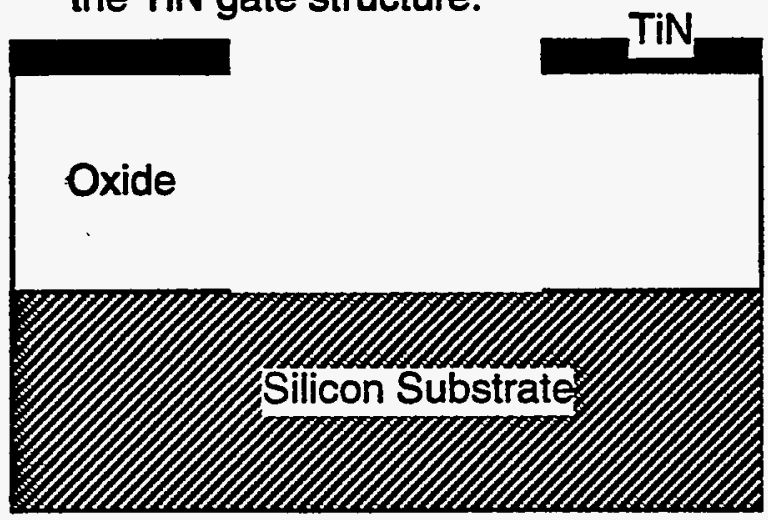

Deposit doped poly spacer film.

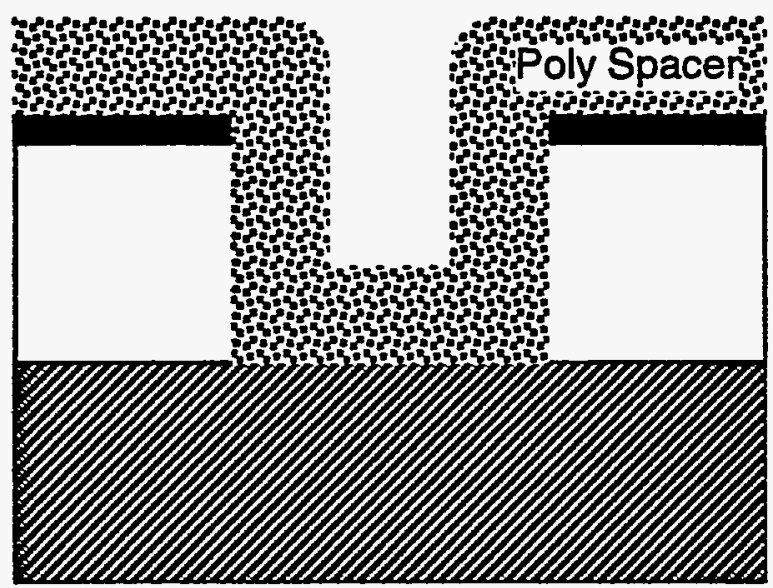

Sputter TiN emitter followed by CVD W deposition

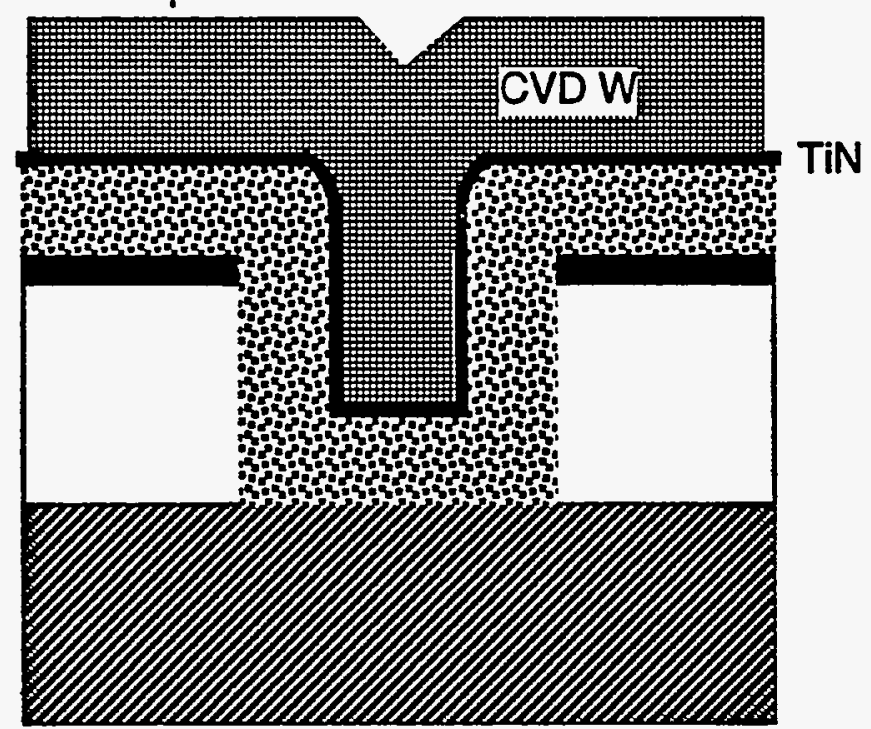

Chemical Mechanical Polish the W/TiN down to the poly layer.

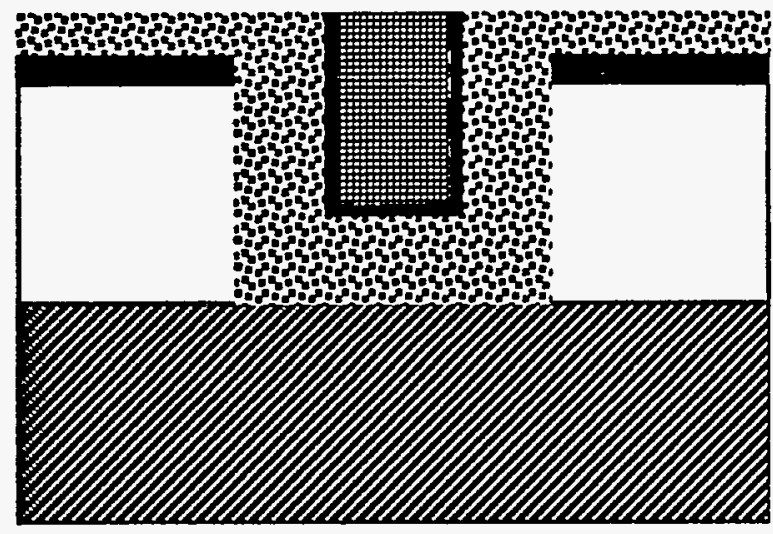

Using a $F$ based reactive ion etching chemistry recess the poly and $W$. TiN is not etched in $\mathrm{F}$ and the resulting "Homs" are the emitter.

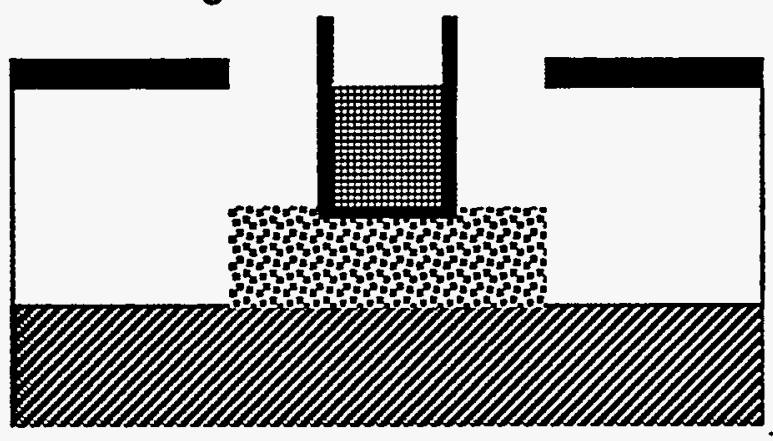

Use a HF wet etch to recess the oxide, in some cases $\mathrm{Ni}$ was also deposited using an evaporator after the oxide etch.

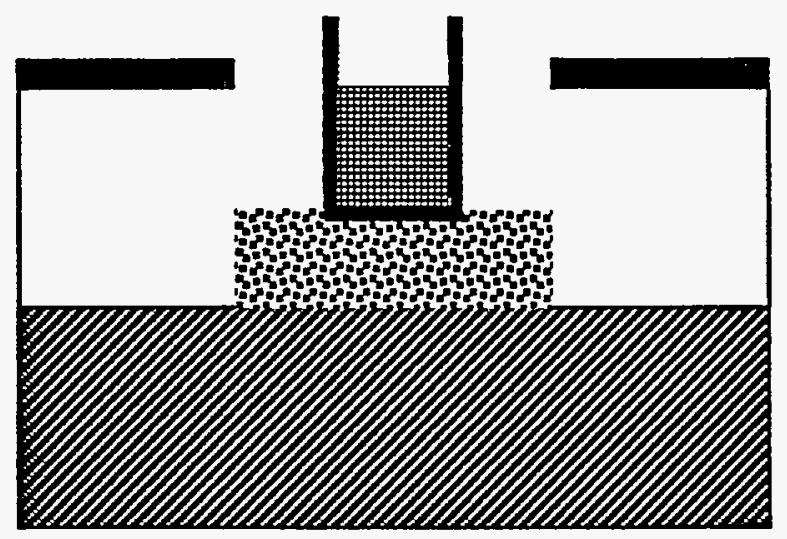




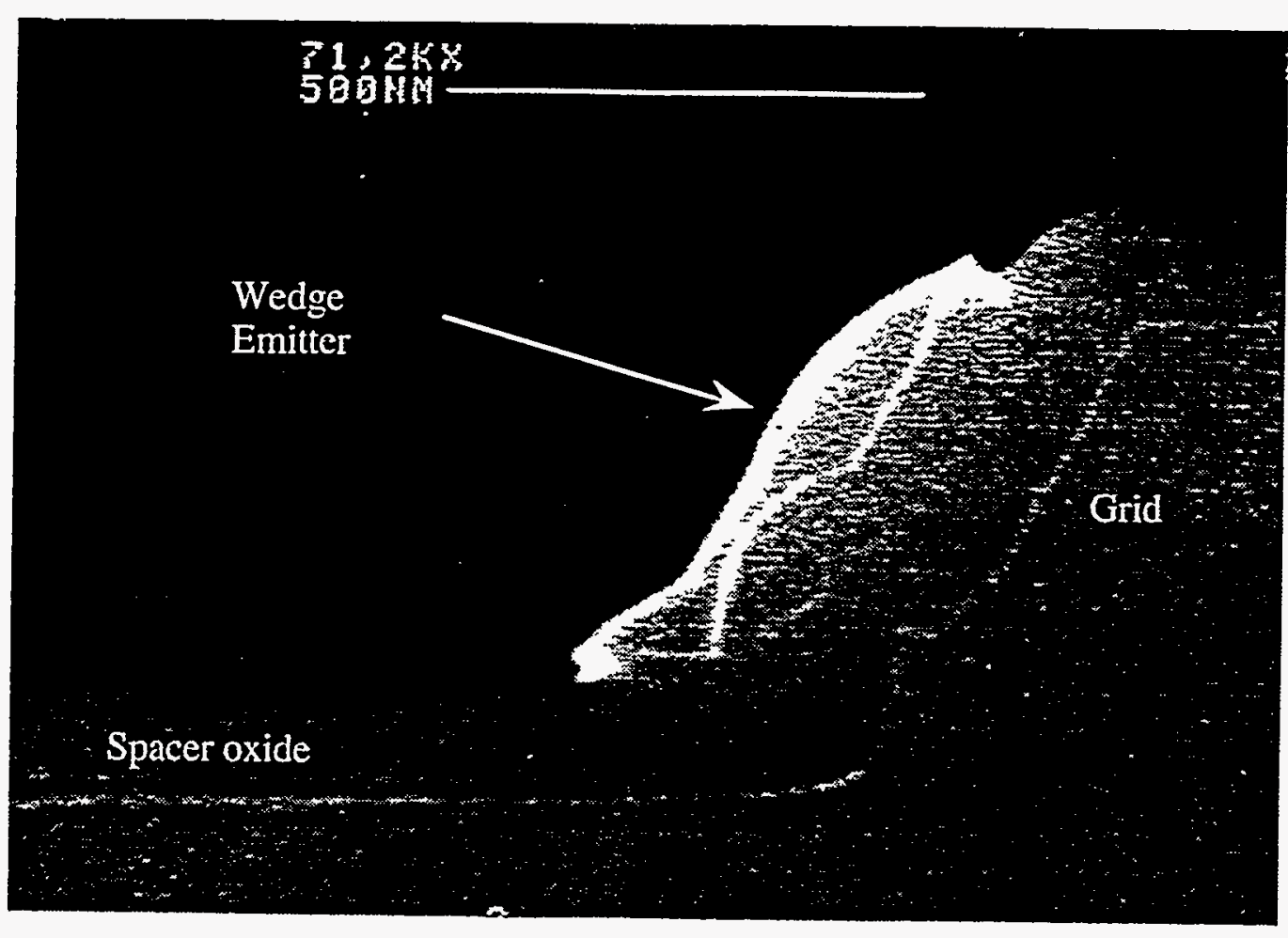

\section{Scanning electron micrograph of a cross section through a device just prior to the final oxide etch step.}

Figure 9

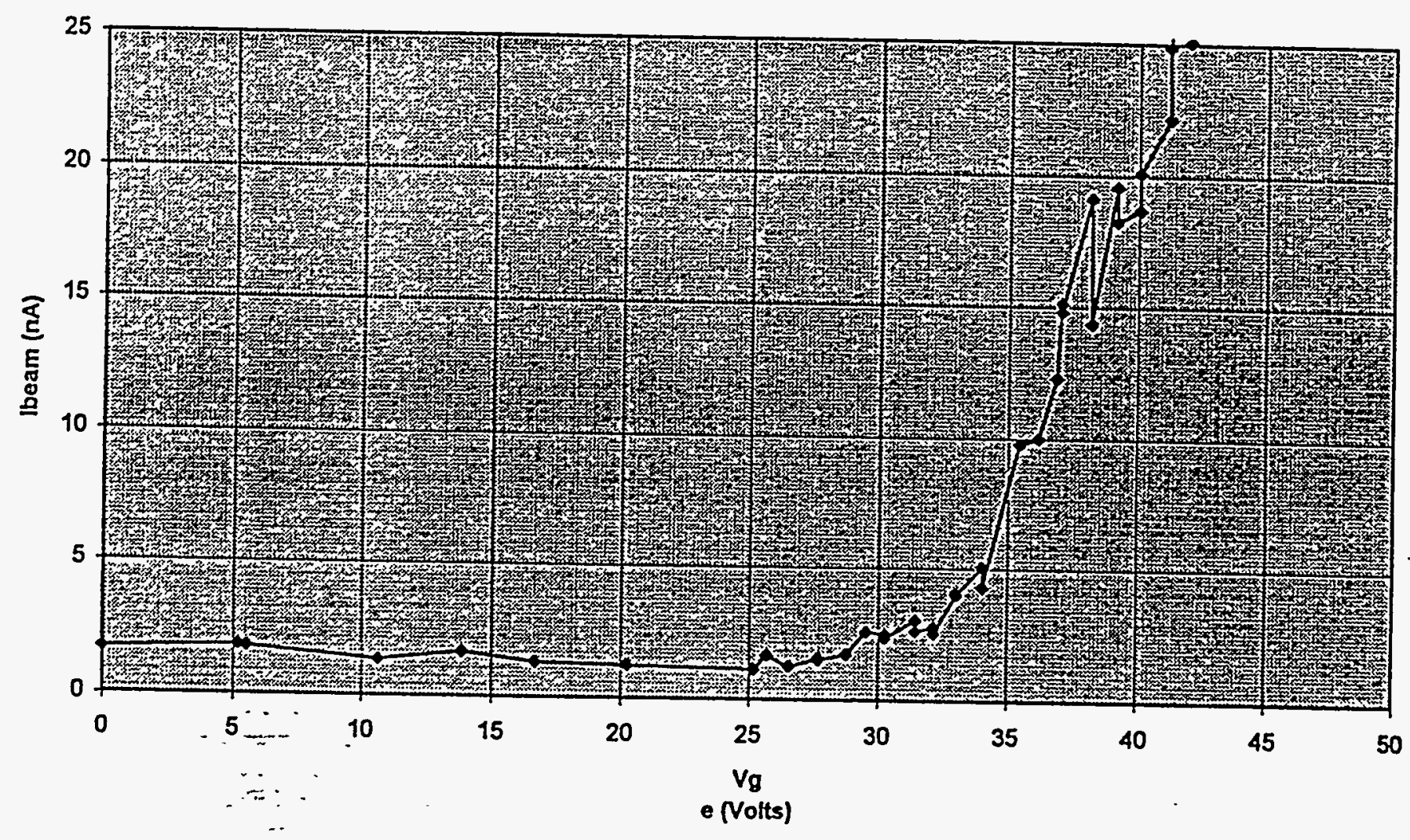

Figure 10 


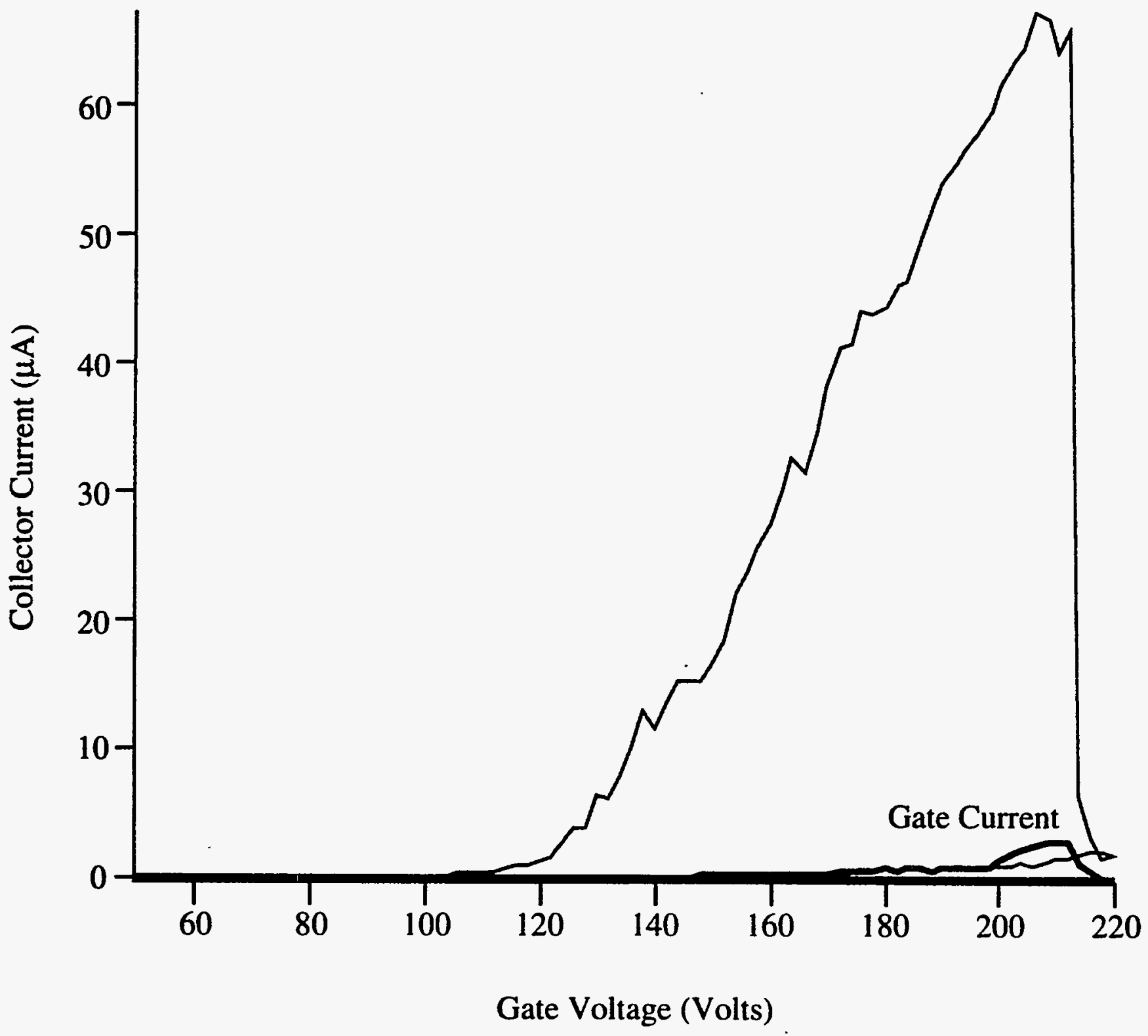

Figure 11 
Distribution:

5 MS1186 Barry Marder, 9541

11153 Collins Clark, 9548

11084 Jim Fleming, 1323

10527 Robert Walko, 1231

11436 C. E. Meyers (LDRD Office), 4503

19017 Central Technical Files, 8523-2

50899 Technical Library, 13414

10619 Print Media, 12616

20100 Document Processing, 7613-2

For DOE/OSTI 




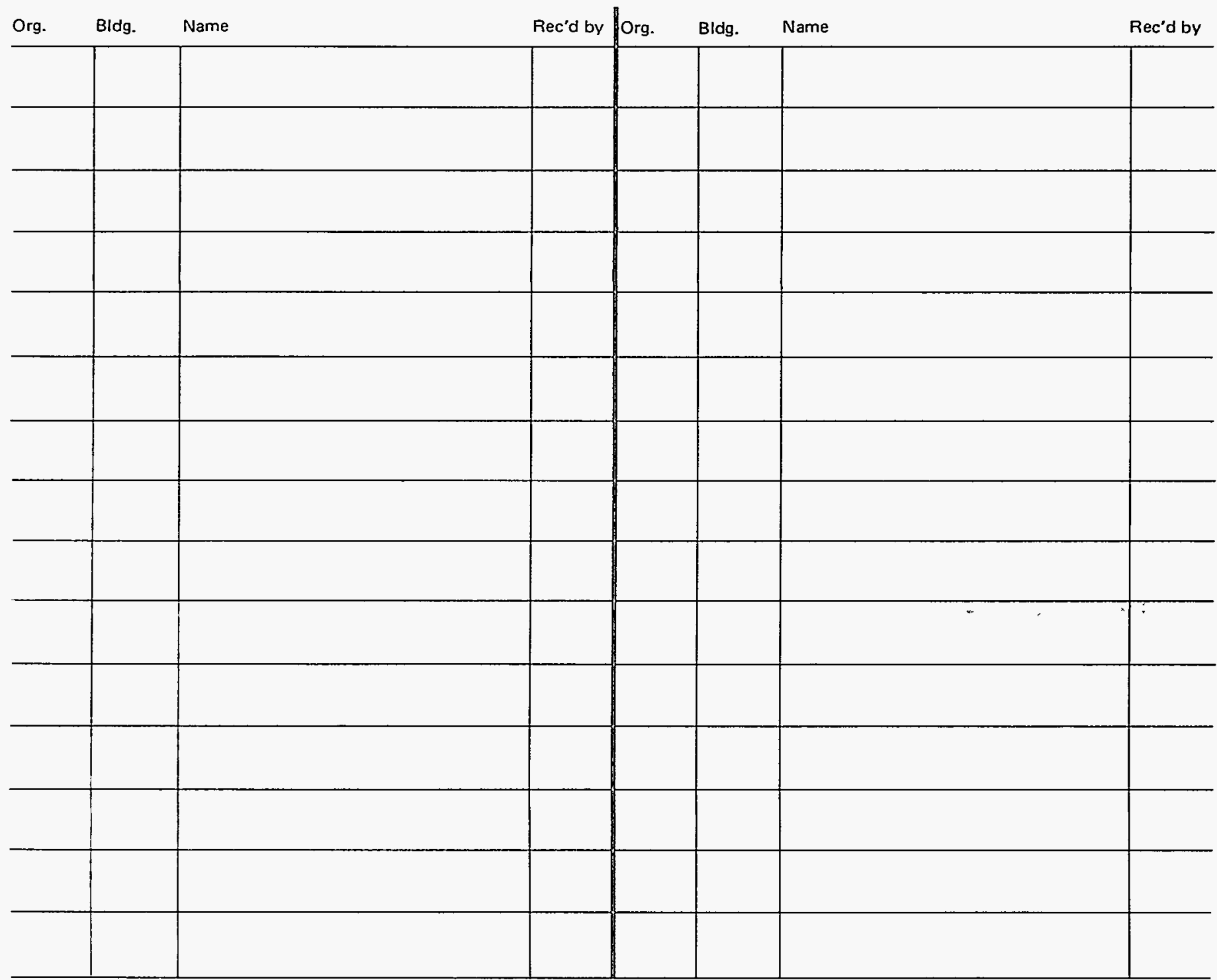

(圆) Sandia Alational Laboratories 